Caballero García, M.F., Alavés-González, V. \& Soler, L. (2020). Influencia de la orientación del espacio sobre el esfuerzo percibido en Educación Física: Juegos motores de cooperaciónoposición. Revista Electrónica Interuniversitaria de Formación del Profesorado, 23(3), 233-247.

DOI: https://doi.org/10.6018/reifop.402151

\title{
Influencia de la orientación del espacio sobre el esfuerzo percibido en Educación Física: Juegos motores de cooperación- oposición
}

\author{
María Fuensanta Caballero García, Verónica Alavés-González, Lorena Soler Mesa \\ Universidad de Murcia
}

\section{Resumen}

Según la Organización Mundial de la Salud (WHO, 2010), en edad infantil, se debe realizar una práctica física mínima de 60 minutos diarios en un rango de intensidad de moderada a vigorosa. Se ha realizado un estudio empírico con 28 alumnos de $6^{\circ}$ de Educación Primaria y se han desarrollado 3 juegos motores de cooperación-oposición, variando la orientación del espacio. Los objetivos de este trabajo han sido conocer la adecuación de esfuerzo percibido en los tres juegos, en relación con la intensidad, para la consecución de beneficios para la salud y comparar el esfuerzo percibido en función de las distintas orientaciones del espacio. Tras la finalización de cada juego, se administró la escala de esfuerzo percibido Pictorical Children's Effort Rating Table (PCERT). Los resultados muestran que los alumnos no alcanzan una intensidad suficiente en los juegos motores para obtener beneficios para la salud, así como diferencias estadísticamente significativas para el esfuerzo percibido en función de la orientación del espacio, siendo el juego con mayor intensidad el que presenta espacio orientado y porterías pequeñas y el juego con menor percepción del esfuerzo es el de espacio no orientado.

\section{Palabras clave}

Educación Física; esfuerzo percibido; orientación del espacio; juegos motores. 


\title{
Influence of space orientation on perceived exertion in Physical Education: Cooperation-opposition motor games
}

\begin{abstract}
According to the World Health Organization (WHO, 2010), as a child, a minimum physical practice of 60 minutes per day should be carried out in a range of moderate to vigorous intensity. An empirical study has been carried out with 28 students of 6th Primary Education and 3 cooperation-opposition motor games have been developed, varying the orientation of the space. The objectives of this work have been to know the adequacy of perceived effort in the three games, in relation to intensity, to achieve health benefits and compare the perceived effort based on the different orientations of the space. After the completion of each game, the Pictorical Children's Effort Rating Table (PCERT). Results show that the students do not reach a sufficient intensity in motor games to obtain health benefits, as well as statistically significant differences for the perceived exertion depending on the orientation of the space, being the game with higher intensity the one that presents oriented space and small goals and the game with less perceived exertion is the non-oriented space game.
\end{abstract}

\section{Key words}

Physical Education, intensity, perceived exertion, space orientation, motor games

\section{Introducción}

\section{Importancia de actividad físico-deportiva infantil}

En la actualidad, como consecuencia de los cambios en los estilos de vida de la población infantil, se hace necesario el estudio de los beneficios que ofrece la actividad física y, específicamente, la actividad física desarrollada en el área de Educación Física (en adelante, EF) para la salud en edades tempranas durante la etapa de Educación Primaria (Sierra, 2003). Así, la gran prevalencia de estilos de vida sedentarios, tanto en edad adulta como en edad infantil, es considerada como un alarmante problema de salud pública. (Blair, 2009).

Como medida de prevención de los efectos negativos del sedentarismo sobre la salud cardiovascular de los niños, la Organización Mundial de la Salud (WHO, 2010) ha desarrollado las recomendaciones mundiales de actividad física para salud en edad infantil, las cuales establecen que la actividad física practicada con regularidad reduce el riesgo de enfermedades cardiovasculares $y$, además, ayuda a conseguir un equilibrio energético controlando el peso. El conjunto de sus investigaciones indica que se debe emplear una práctica física moderada o vigorosa durante un mínimo de 60 minutos diarios para mejorar la salud y prevenir las enfermedades no transmisibles. Además, Stratton (1997), determina que la intensidad adecuada de las sesiones de Educación Física en relación con la salud es aquélla que se encuentra en valores de más de 150 pulsaciones por minuto.

No obstante, en edad infantil, son varios los estudios que determinan que los niños no son suficientemente activos. Según Laguna, Lara y Aznar (2011) la población española presenta una condición física inferior en comparación con otros países, debido a su baja participación 
en actividades físicas. Además, la mayoría de los niños no realizan suficiente actividad física para llegar a tener beneficios para la salud. Según Montil, Barriopedro y Oliván (2005), sólo un 20\% de los niños de 10 a 13 años realiza una hora de actividad física diaria, un 79'1\% media hora, al menos 5 días a la semana y el resto no lo supera. Por lo que destaca el no cumplimiento de las recomendaciones, debiéndose a la frecuencia de práctica y no al tiempo de la misma.

Para el correcto tratamiento de esta problemática se requiere un enfoque multidisciplinar, en el cual se tienda a modificar hábitos nutricionales y de vida del niño y de su entorno familiar y social (Lobos, Leyton, Kain y Vio, 2013). En la etapa de educación obligatoria, la Educación Física es considerada como un área que podría contribuir a la realización de actividad física y a la prevención del sobrepeso y la obesidad. Además, la Educación Física puede llegar a los niños y adolescentes, los cuales son el futuro de nuestra sociedad y se encuentran en una etapa fundamental para adquirir y consolidar hábitos y un estilo de vida saludable y activo (Biddle, Sallis y Cavill, 1998).

Para llevar a cabo esta tarea de promoción de la actividad física orientada a la salud, la escuela es el lugar idóneo (Garrido, 2014). La Educación Física es una potente herramienta para dotar a las personas de habilidades, conocimientos y actitudes para realizar ciertas acciones en confluencia de una vida más saludable (González-Arévalo y Lleixà-Arribas, 2010). El colegio tiene una significativa participación en la actividad metabólica del niño ya que gran parte de la población infantojuvenil la actividad física que practica es la que se lleva a cabo fundamentalmente durante su estancia en la escuela y no en el tiempo de ocio (Villagrán, Rodríguez-Martín, Novalbos, Martínez y Lechuga, 2010).

Varios estudios demuestran que las clases de Educación Física ayudan en el alumnado de primaria a incrementar la actividad física moderada-vigorosa, respecto a la actividad física que realizan durante la semana (Sanchís, Calahorro, Lara y Torres, 2015) así como que los centros educativos no contribuyen para la consecución de un estilo de vida saludable (González, Fraguela y Varela, 2017; Tuñón y Laíno, 2014).

No obstante, también son varios los estudios que demuestran que la Educación Física en nuestro país es insuficiente en términos de frecuencia e intensidad. Según Gambau (2015) los datos aportados por la Comisión Europea muestran que España ocupa uno de los últimos puestos en horas lectivas empleadas en Educación Física, hecho que, añadiendo la falta de coordinación con la práctica deportiva extracurricular de los alumnos, hace que nuestro país actúe con modelos deportivos anacrónicos propios del siglo XX.

Del mismo modo, el estudio realizado por Albarracín, Moreno y Beltrán (2014), muestra la situación actual de la Educación Física según su profesorado en la Región de Murcia. Los docentes piensan que esta área no tiene suficiente carga horaria y contrastado con la práctica de algunos profesionales, consideran que acaban impartiendo siempre los mismos contenidos. Por este motivo, se destaca que actualmente la E.F es un área de estatus bajo y con escasa valoración por parte de la comunidad educativa, dejando a esta en una situación preocupante.

Para finalizar y profundizando más aún en este problema, otros estudios previos han determinado que la intensidad de las clases de Educación Física tampoco es suficiente para la consecución de beneficios sobre la salud, es decir, durante el desarrollo de las mismas no se alcanza un rango de moderada a vigorosa intensidad, tal y como recomiendan las 
instituciones mundiales (McKenzie, Marshall., Sallis y Conway, 2000; Nader y NICHD, 2003; Simons-Morton, Taylor, Zinder y Huang, 1993).

\section{Efecto de la orientación del espacio en la intensidad}

El juego motor es el principal recurso del que dispone el área de E.F para el desarrollo de los contenidos de enseñanza-aprendizaje (Baena y Ruiz, 2016). En este sentido, es a través de la modificación de los elementos estructurales de las situaciones motrices o juegos motores como se puede llegar a alcanzar los valores adecuados de intensidad para la edad infantil en las sesiones de Educación Física. Para ello, siguiendo la ciencia de la acción motriz, denominada Praxiología Motriz (Parlebas, 2001), se hace necesaria la modificación de los elementos estructurales que componen la lógica interna de las situaciones motrices. La lógica interna se entiende como el conjunto de relaciones internas que demanda un juego y sus consecuencias sobre la práctica. Así, la lógica interna está formada por elementos estructurales, tales como el espacio, el tiempo, los materiales y las relaciones entre participantes. Sus características pueden variar para poder constatar el efecto de los mismos sobre las intensidades que se alcanzan en las sesiones. Según el orden que exija la lógica interna puede hacer que los jugadores se relacionen de manera determinada (colaborando $u$ oponiéndose), utilizando espacios concretos, manipulando el material de forma precisa, respetando las secuencias temporales e iniciand y y finalizando el juego de maneras prefijadas (Lavega, 2007).

Si se tiene en cuenta la importancia de los elementos estructurales de los juegos motores, la orientación del espacio debería ser considerada como un factor influyente en la carga interna de los juegos motores y, en definitiva, de la intensidad alcanzada en los mismos (Casamichana, Castellano, González-Morán, García-Cueto y García-López, 2011). Cabe señalar que el término orientación del espacio hacer referencia a la presencia o no presencia de objetivos en el espacio donde conseguir interacciones de marca (Parlebas, 2001), como, por ejemplo, es el establecimiento de porterías en un juego donde el juego es orientado para anotar los tantos hacia ese espacio. La orientación del espacio debe ser un aspecto a considerar por los maestros de Educación Física debido a la influencia que este factor puede causar sobre la variable de intensidad durante la práctica de actividad física (Mallo y Navarro, 2008).

\section{El esfuerzo percibido como indicador de intensidad}

Uno de los principales parámetros que determinan la idoneidad de las sesiones de Educación Física es la intensidad del esfuerzo percibido. A fin de valorar intensidad en Educación Física, se utiliza la percepción subjetiva del esfuerzo o Rating of Perceived Exertion (RPE). Este es un indicador válido para su medición (Naclerio, Barriopedro y Rodríguez, 2008; Som, Zabala, Sánchez-Sánchez, Sánchez-Muñoz y Ramírez, 2009). El valor de la percepción subjetiva del esfuerzo reside en la relación existente entre los valores de dicha percepción con el porcentaje de la frecuencia cardíaca máxima, el porcentaje del consumo máximo de oxígeno y la concentración de lactato en sangre, lo que hace que los datos sobre este indicador sean fundamentales para el conocimiento de la intensidad durante las sesiones (Coquart et al., 2009; Nacleiro, Barriopedro y Rodíguez, 2009).

Existen varias escalas que son utilizadas para medir el esfuerzo percibido durante la práctica de ejercicio. No obstante, la escala Borg o escala de Percepción Subjetiva del Esfuerzo (Borg, 1982 citado en Ozkan y Kin-Isler, 2007) es utilizada como uno de los mejores instrumentos para medir el grado de esfuerzo físico. A pesar de que la escala de Borg es la más comúnmente utilizada, en los últimos años se han creado otras escalas, entre la que se 
encuentra, por ejemplo, la escala OMNI, de la cual se ha probado su validez (Utter et al., 2004).

No obstante, las escalas citadas anteriormente han sido validadas para hallar los datos de esfuerzo percibido en personas mayores de 12 años, por lo que se han creado instrumentos adecuados para medir el esfuerzo percibido en escolares de 6 a 12 años de edad. De este modo, han surgido escalas validadas para participantes de edades tempranas como es la escala PCERT o Pictorical Children's Effort Rating Table de Yelling, Lamb, Swaine (2002). Así, la escala PCERT fue diseñada para niños y fue validada por Yelling et al. (2002) usando el ritmo cardíaco. La relación entre la percepción del esfuerzo y el ritmo cardíaco obtuvo una alta fiabilidad, correspondiéndose los valores 4 y 5 de la escala PCERT con 140 y 150 pulsaciones por minuto como valor promedio (Kang, Hoffman, Walker, Chaloupka y Utter, 2003).

En definitiva, son diversos los estudios que han utilizado las escalas de esfuerzo percibido para determinar la intensidad de las sesiones de actividad física (Hernández-Álvarez et al., 2010; Lambrick, Bertelsen, Eston, Stoner y Faulkner, 2016), así como la utilización de estas escalas como un procedimiento práctico para poder controlar la intensidad durante la actividad física (De Hoyo y Sañudo, 2007)

\section{Objetivos}

Por todo lo expuesto anteriormente, los objetivos generales de este trabajo son los siguientes:

1. Analizar la adecuación del esfuerzo percibido en cada uno de los tres juegos motores de cooperación-oposición, con distintas orientaciones del espacio, para la consecución de beneficios para la salud.

2. Comparar la intensidad mediante los niveles de esfuerzo percibido, en función de las distintas orientaciones del espacio, de los juegos motores de cooperación-oposición.

\section{Material y método}

\section{Participantes}

Los participantes fueron 28 estudiantes, pertenecientes a $6^{\circ}$ de Educación Primaria. El rango de edad de los participantes fue de 10 a 12 años ( $N=13$ niños, Edad: $\bar{x}=10,85$, DT=0,55; $N=15$ niñas, Edad: $\bar{x}=11,00, \mathrm{DT}=0,37)$. Los padres o tutores legales fueron informados del procedimiento de la investigación mediante una hoja de información detallada y firmaron un consentimiento informado previo a la realización de la investigación.

\section{Procedimiento}

Para la realización del estudio, se estableció como variable independiente la orientación del espacio. De esta manera, se analiza el esfuerzo percibido de tres juegos motores de cooperación-oposición para trabajar el pase y el desmarque, variando la orientación de los mismos: un juego con espacio no orientado o de mantenimiento, un juego con espacio orientado y porterías grandes y un juego con espacio orientado y porterías pequeñas (Figura 1). Todos los juegos se desarrollaron en un espacio de $20 \times 20 \mathrm{~m}$. 


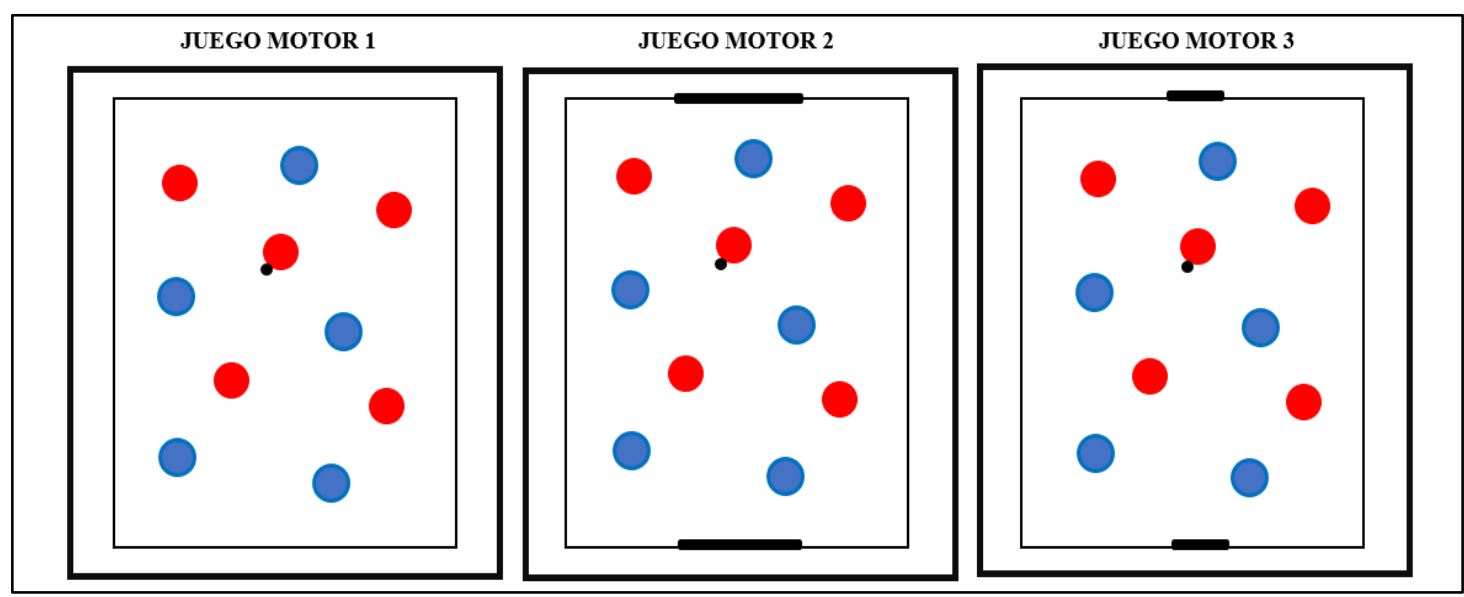

Figura 1. Representación gráfica de los juegos motores (tamaño de $20 \times 20$ metros)

Para la valoración de la intensidad durante cada juego se utilizó la escala de esfuerzo percibido Pictorical Children's Effort Rating Table (PCERT), validada por Yelling et al. (2002). Al finalizar cada juego, se establece un periodo de 5 minutos que sirve de descanso entre juegos y para cumplimentar el instrumento de esfuerzo percibido en la zona establecida para ello. Cabe señalar que previo a la realización de este estudio los participantes realizaron un periodo de familiarización, durante 4 sesiones previas, con la escala PCERT de esfuerzo percibido.

Siguiendo las conclusiones de Stratton (1997), se determina que los alumnos que llegan a una intensidad superior de 150 pulsaciones por minuto, se encuentran en valores óptimos para la consecución de beneficios para la salud. Para alcanzar intensidades superiores a 150 pulsaciones por minuto, se han considerado los valores iguales o superiores a 5 en la escala PCERT, pues, según el estudio de Kang et al. (2003), este valor se corresponde con 150 pulsaciones por minuto. De este modo, se establece que el porcentaje de alumnos que debe encontrarse en valores iguales o superiores a 5 en la escala PCERT para que el juego sea considerado óptimo debe ser igual o superior al $85 \%$ de los participantes.

\section{Instrumento}

Para la toma de datos de esfuerzo percibido se se utilizó la escala Pictorical Children's Effort Rating Table (PCERT) elaborada y validada por Yelling, Lamb y Swaine (2002) (Figura 2). Además, a los participantes se les entregaba una ficha individual, donde rellenar los datos de las tres actuaciones puntuales (tres juegos de cooperación-oposición).

\section{Análisis de datos}

Se realizaron estadísticos descriptivos (mínimo, máximo, media y desviación típica) para el esfuerzo percibido en los tres juegos de cooperación-oposición. Del mismo modo, se han realizado porcentajes para cada uno de los juegos en función de la cantidad de alumnos que alcanzaba el nivel mínimo de esfuerzo percibido.

El supuesto de normalidad para el esfuerzo percibido fue realizado mediante la prueba Shapiro-Wilk. Tras comprobar la normalidad para el esfuerzo percibido de los dos juegos con espacios orientados y la no normalidad del juego sin espacio orientado, se han realizado las pruebas de contraste de medias T de Student y W de Wilconxon. El tamaño del efecto se ha calculado mediante la prueba $d$ de Cohen (efecto pequeño $=0,20$; efecto moderado $=0,50$ y 
efecto grande=0,80). Se ha establecido un valor de significación $\mathrm{p} \leq .0,05$. Para el tratamiento estadístico se ha utilizado el programa SPSS V.17.0.

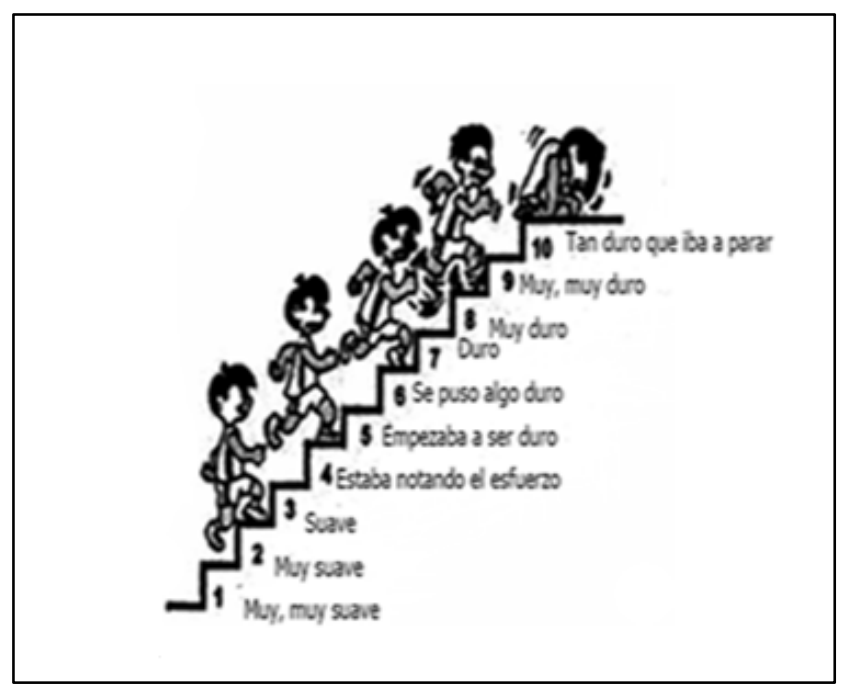

Figura 2. Escala de esfuerzo percibido Pictorical Children's Effort Rating Table (PCERT)

\section{Resultados}

\section{Resultados para el primer objetivo de estudio}

Teniendo en cuenta el primer objetivo del estudio, se pretende analizar la adecuación de los tres juegos motores, con distintas orientaciones del espacio para la consecución de beneficios para la salud, en función de las recomendaciones establecidas por la WHO (2010).

Siguiendo los límites establecidos por Stratton (1997), los estadísticos descriptivos (porcentajes) determinan la cantidad de alumnos que se encuentran dentro de los valores adecuados, es decir, más de 150 pulsaciones por minuto, lo que se corresponde con valores superiores al nivel 5 en la escala PCERT de esfuerzo percibido.

Los resultados descriptivos (Tabla 1) muestran que la media para la media para el primer juego desarrollado ha sido de $\bar{x}=2.25 \pm 1.20$, mientras que del juego con espacio orientado y porterías grandes la media ha sido de $\mathrm{x} \pm 1.73$ y la media para el juego con espacio orientado $\mathrm{y}$ porterías pequeñas ha sido de pequeñas $\bar{x}=5.11 \pm 2.23$.

Para el primer juego desarrollado, el cual no presenta orientación del espacio, los porcentajes (Figura 3) determinan que solamente el $4 \%$ de los alumnos se encuentran en valores de esfuerzo percibido óptimos, estando el $96 \%$ restante de los alumnos en rangos de intensidad inadecuados para la obtención de beneficios para la salud. Se muestra que, para el juego sin espacio orientado o de mantenimiento, el porcentaje de participantes que alcanza los valores óptimos no llega al porcentaje de $85 \%$ mínimo establecido para considerar adecuado el juego para obtener beneficios sobre la salud. 
Tabla 1.

Estadísticos descriptivos para los valores de esfuerzo percibido en los tres juegos

\begin{tabular}{lllllll}
\hline & & N & Mínimo & Máximo & Media & Desv. típ. \\
\hline $\begin{array}{l}\text { Juego sin orientación del } \\
\text { espacio }\end{array}$ & 28 & 1 & 5 & 2.25 & 1.20 \\
\hline $\begin{array}{l}\text { Juego con orientación del } \\
\text { espacio (porterías grandes) }\end{array}$ & 28 & 1 & 8 & 3.86 & 1.73 \\
\hline $\begin{array}{l}\text { Juego con orientación del } \\
\text { espacio (porterías pequeñas) }\end{array}$ & 28 & 1 & 9 & 5.11 & 2.23 \\
\hline
\end{tabular}

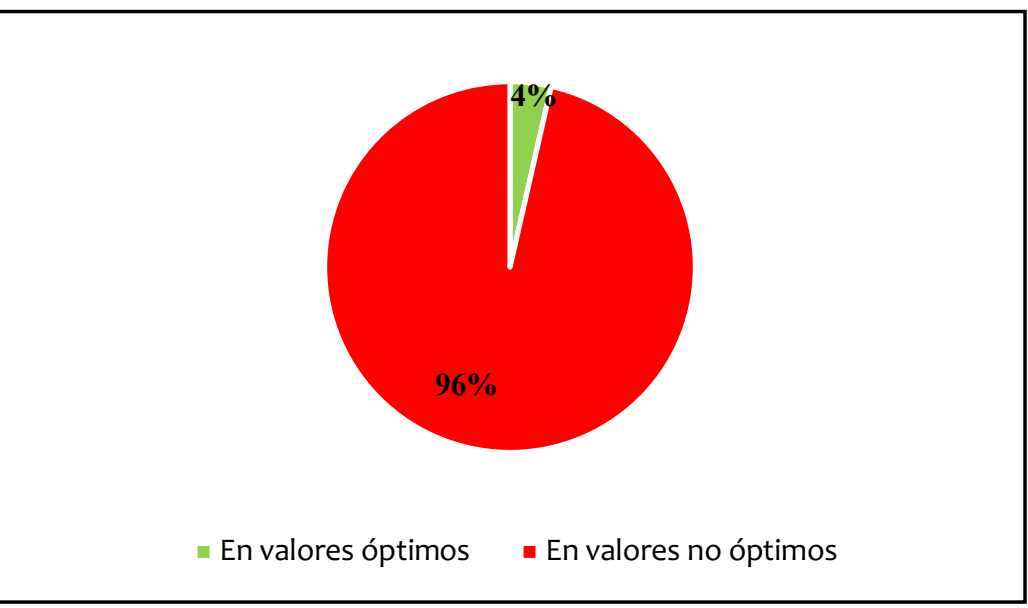

Figura 3. Porcentajes de alumnado en valores óptimos y en valores no óptimos para la obtención de beneficios en el Juego 1 (sin orientación del espacio)

Para el segundo juego motor, que presenta orientación del espacio hacia cuatro porterías, los porcentajes (Figura 4) muestran que solamente el $32 \%$ de los alumnos se encuentran en valores de esfuerzo percibido óptimos, estando el $68 \%$ de los alumnos en rangos de intensidad inadecuados para la obtención de beneficios para la salud. Se muestra que, para el juego de espacio orientado con porterías grandes, el porcentaje de participantes que alcanza los valores óptimos no llega al porcentaje de $85 \%$ establecido para considerar adecuado para la obtención de beneficios sobre la salud.

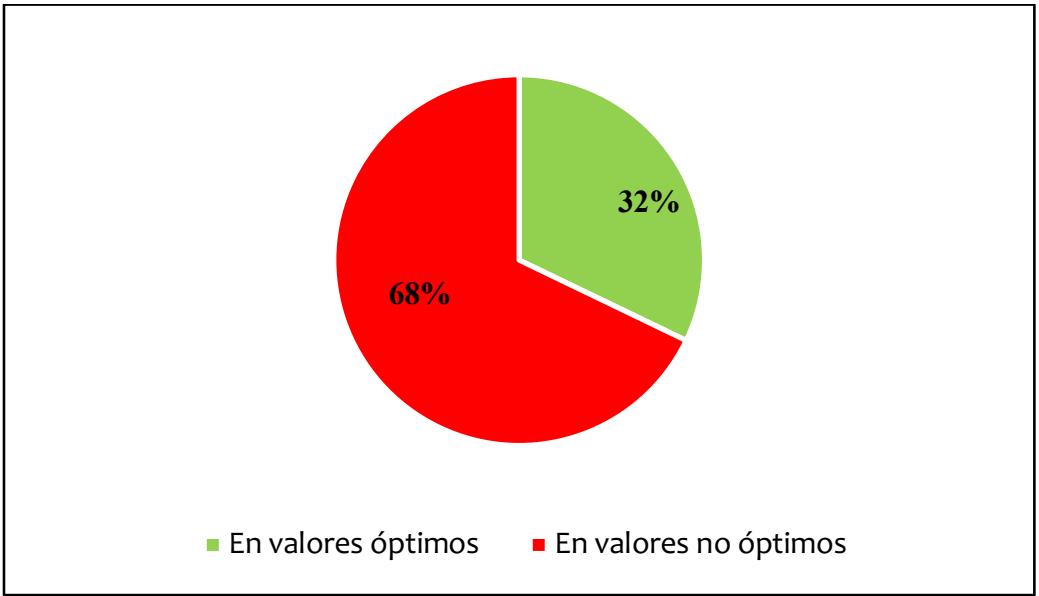

Figura 4. Porcentajes de alumnado en valores óptimos y en valores no óptimos para la obtención de beneficios en el Juego 2 (Espacio orientado con porterías grandes) 
Para el tercer juego desarrollado, el cual presenta orientación del espacio hacia dos porterías, los porcentajes (Figura 5) determinan que el 61\% de los alumnos se encuentran en valores de esfuerzo percibido óptimos, estando el 39\% restante de los alumnos en rangos de intensidad inadecuados para la obtención de beneficios para la salud. Se muestra que, para el juego de espacio orientado con porterías pequeñas, el porcentaje de participantes que alcanza los valores óptimos no llega al porcentaje de $85 \%$ mínimo establecido para considerar adecuado el juego para obtener beneficios sobre la salud.

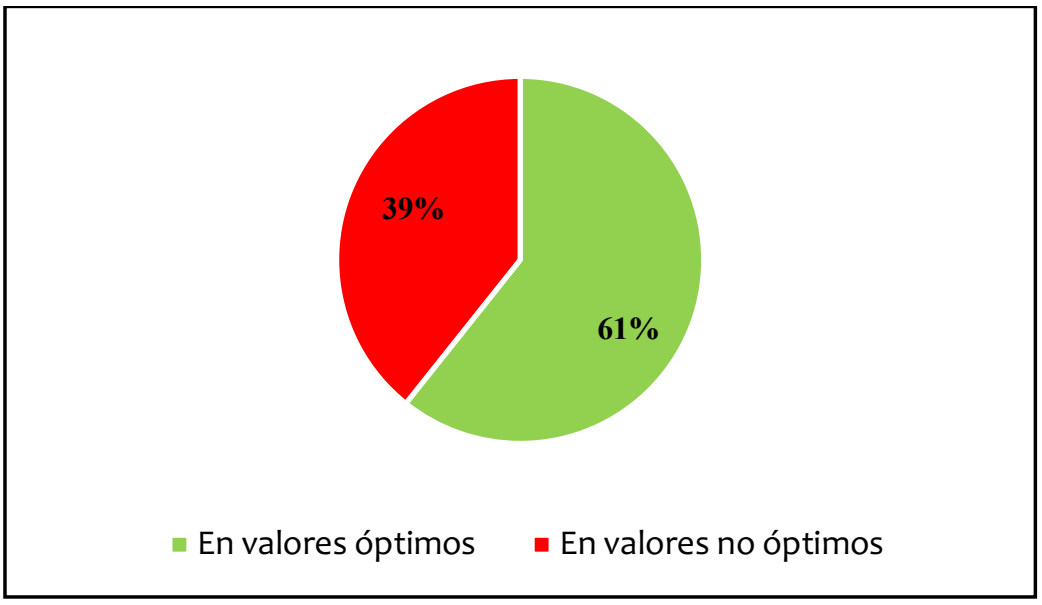

Figura 5. Porcentajes de alumnado en valores óptimos y en valores no óptimos para la obtención de beneficios en el Juego 3 (Espacio orientado con porterías pequeñas)

\section{Resultados para el segundo objetivo de estudio}

Teniendo en cuenta el segundo objetivo de la investigación, el cual hace referencia a comparar los niveles de esfuerzo percibido en función de las distintas orientaciones del espacio de los juegos motores de cooperación-oposición, se presentan los resultados de comparación de medias para el esfuerzo percibido en cada uno de los tres juegos.

Los resultados de las pruebas de comparación de medias (Tabla 2) determinan diferencias estadísticamente significativas para cada uno de los tres juegos respecto a los dos restantes.

Así, tal y como se observa, se ha determinado que el esfuerzo percibido y, por tanto, la intensidad del primer juego de cooperación-oposición, ha sido significativamente menor que en los otros dos juegos $(p<0,05)$.

Tabla 2.

Pruebas de comparación de medias para el esfuerzo percibido entre los tres juegos desarrollados

\begin{tabular}{|c|c|c|c|}
\hline & JUEGOS & $\mathrm{p}$ & d \\
\hline \multirow{2}{*}{ Par 1} & Juego sin orientación del espacio & \multirow{2}{*}{0.00} & \multirow{2}{*}{1.08} \\
\hline & Juego con orientación del espacio (porterías grandes) & & \\
\hline \multirow{2}{*}{ Par 2} & Juego sin orientación del espacio & \multirow{2}{*}{0.00} & \multirow{2}{*}{1.59} \\
\hline & Juego con orientación del espacio (porterías pequeñas) & & \\
\hline \multirow{2}{*}{ Par 3} & Juego con orientación del espacio (porterías grandes) & \multirow{2}{*}{0.02} & \multirow{2}{*}{0.62} \\
\hline & Juego con orientación del espacio (porterías pequeñas) & & \\
\hline
\end{tabular}


Se determina que el esfuerzo percibido en el juego sin orientación del espacio ( $\bar{x}=2.25 \pm 1.20$ ) ha sido significativamente menor que el esfuerzo percibido en el juego con una orientación del espacio con porterías grandes ( $\bar{x}=3.86 \pm 1.737)$, con un tamaño del efecto grande.

Del mismo modo, el esfuerzo percibido en el juego sin orientación del espacio ( $\bar{x}=2.25 \pm 1.20$ ) ha sido significativamente menor que el esfuerzo percibido en el juego con espacio orientado y con porterías grandes $(\bar{x}=5.11 \pm 2.23)$, con un tamaño del efecto grande.

A su vez, tal y como se presenta en la tabla 3 , se han determinado diferencias estadísticamente significativas $(p<0.05)$ entre el juego con espacio orientado y con porterías grandes $(\bar{x}=3.86 \pm 1.737)$ y el juego con orientación del espacio y con porterías pequeñas $(\bar{x}=5.11 \pm 2.23)$, con un tamaño del efecto medio.

En definitiva, el juego que más intensidad del esfuerzo ha generado ha sido el tercer juego, con espacio orientado hacia porterías pequeñas, seguido del segundo juego, con orientación del espacio hacia porterías grandes. Y, por último, el juego que menos esfuerzo percibido ha generado ha sido el primer juego, el cual no presentaba orientación del espacio y que consistía en un juego de mantenimiento del balón, siendo menor el esfuerzo percibido y, por ende, la intensidad en el juego motor sin orientación del espacio respecto a los dos juegos motores con el espacio orientado.

\section{Discusión}

\section{Discusión para el primer objetivo de estudio}

El primer objetivo de este trabajo ha sido analizar la adecuación del esfuerzo percibido en cada uno de los tres juegos de cooperación-oposición, con distintas orientaciones del espacio, para la consecución de beneficios para la salud.

Siguiendo las recomendaciones establecidas por la WHO (2010), las sesiones de Educación Física, deben generar una intensidad de la práctica en un rango de moderada a vigorosa intensidad. La literatura científica también establece que las pulsaciones por minuto que los estudiantes deben alcanzar en las sesiones de Educación Física debe ser mayores 150 pulsaciones por minuto (Stratton, 1997), lo que se corresponde con un valor igual o superior a 5 en la escala PCERT (Kang et al., 2003). De este modo, teniendo en cuenta que las sesiones de Educación Física se componen de las distintas situaciones motrices desarrolladas en ellas, es necesario que cada situación motriz o juego motor alcance los valores mínimos establecidos.

Teniendo en cuenta dichos valores, dos de los juegos desarrollados en el estudio, el juego con espacio no orientado y el juego con espacio orientado y porterías grandes, no han alcanzado la intensidad óptima de 150 pulsaciones por minuto, siendo lo valores de esfuerzo percibido muy bajos, con 2,25 y 3,86 puntos de media respectivamente. Así, se puede verificar que en ninguno de estos juegos se ha llegado a alcanzar incluso las 140 pulsaciones por minuto. Además, los resultados de la investigación han determinado que los porcentajes de alumnos que se encuentran en valores óptimos para la salud en ambos juegos han sido reducidos, siendo del $4 \%$ para el juego sin espacio orientado o de mantenimiento y del $32 \%$ en el juego con espacio orientado y porterías grandes; porcentajes inferiores al $85 \%$ establecido como mínimo de alumnos que debieran obtener valores superiores a 5 en la escala de esfuerzo percibido para considerar el juego como adecuado para mejorar la salud. 
Por su parte, el juego con espacio orientado y porterías pequeñas sí ha obtenido un valor medio de esfuerzo percibido ligeramente superior al valor 5 en la escala PCERT de esfuerzo percibido, con una media de 5,11 puntos. De esta manera, se puede corroborar que éste ha sido el único juego motor que ha obtenido un valor medio de esfuerzo percibido adecuado para la consecución de beneficios para la salud. Sin embargo, aunque en este tercer juego motor la media de esfuerzo percibido obtenida ha sido superior al valor 5 , el porcentaje de alumnos respecto al total de participantes que ha alcanzado dicho valor ha sido del $61 \%$, porcentaje alejado del $85 \%$ del total, el cual debiera ser el porcentaje mínimo de alumnos para considerar el juego óptimo para la consecución de beneficios sobre la salud.

Son diversas las investigaciones previas que Han determinado que las sesiones de Educación Física son insuficientes en términos de intensidad que se genera durante el desarrollo de las mismas, resultados que coinciden con los obtenidos en esta investigación. En el estudio realizado por Simons-Morton et al. (1993), se determinó que los estudiantes de Educación Primaria solamente alcanzaban los rangos adecuados de actividad física de moderada a vigorosa intensidad en un $8,5 \%$ del tiempo total de las clases de Educación Física. Del mismo modo, otros estudios han estudiado las zonas de intensidad mediante acelerómetros, determinado que los alumnos de Educación Primaria solamente alcanzaban el rango de actividad física de moderada a vigorosa intensidad durante 9,25\% y 29,4 minutos semanales (Martínez, Contreras, Aznar y Lera, 2012; McKenzie et al., 2000; Nader y NICHD, 2003), duración muy escasa teniendo en cuenta los 60 minutos diarios recomendados en ese rango para la edad de los participantes.

Por todo lo expuesto, si tenemos en cuenta que las sesiones de enseñanza-aprendizaje en el área de Educación Física se componen, en su mayor parte, de situaciones motrices o juegos motores, se puede corroborar que las intensidades alcanzadas en los juegos motores realizados, así como los porcentajes de alumnado respecto al total en el rango adecuado, no parecen mostrar idoneidad de estos juegos motores para alcanzar beneficios sobre la salud.

\section{Discusión para el segundo objetivo de estudio}

El segundo objetivo de la investigación trata de comparar la intensidad mediante los niveles de esfuerzo percibido, en función de las distintas orientaciones del espacio de los juegos motores de cooperación-oposición. Conocer los efectos de las modificaciones de las orientaciones del espacio sobre las intensidades de los juegos motores ofrece una valiosa información a los docentes del área de Educación Física para mejorar y evaluar las sesiones de enseñanza-aprendizaje, en relación a la adecuación de las mismas para la consecución de beneficios sobre la salud de los alumnos.

En este estudio, la variable percepción del esfuerzo, relacionada con la intensidad de las situaciones motrices, ha obtenido diferencias estadísticamente significativas para cada uno de los tres juegos respecto a los dos restantes. Así, el juego motor que ha generado un mayor esfuerzo percibido e intensidad ha sido el de espacio orientado con porterías pequeñas, seguido del juego motor con espacio orientado y porterías grandes y, por último, el juego motor que menos esfuerzo percibido e intensidad ha generado ha sido el de espacio no orientado o de mantenimiento del móvil.

Una posible explicación a la escasa intensidad alcanzada en el juego sin espacio orientado o de mantenimiento del balón podría haber sido la poca demanda de movimiento del juego motor, repercutida por el escaso pensamiento táctico de los alumnos en estas edades para aprovechar los espacios libres en juegos donde no existe una zona específica para alcanzar la meta, en este caso, la inexistencia de porterías. Así, algunos estudios previos que han estudiado el conocimiento táctico de niños en deportes de invasión determinan que, cuando el conocimiento táctico es bajo, existe tendencia de preocupación centralizada en el móvil o 
el balón, por lo que se produce una aglutinación de los jugadores en torno al móvil en un espacio reducido (Bayer, 1992; De la Vega, 2002; Lago, 2002; Martínez, 2007), lo que hace más difícil el desarrollo del juego motor (Lago, 2002) y puede influir en las demandas del movimiento.

Teniendo en cuenta las aportaciones de Casamichana et al. (2011), la posible reducción de las intensidades cuando se orienta el espacio, vendría determinada por la disminución del espacio individual de interacción (Parlebas, 2011), el cual se habría visto disminuido al aumentar el tamaño de las porterías. Así, se constata que el tamaño de las porterías puede influir en el pensamiento táctico de los jugadores, así como en las demandas fisiológicas de los juegos motores.

\section{Conclusiones}

Como conclusiones de la investigación, se verifica que los juegos motores desarrollados no han alcanzado los valores mínimos de esfuerzo percibido para la consecución de beneficios sobre la salud, a excepción del juego con espacio orientado y porterías pequeñas, que ha obtenido un valor ligeramente superior al valor 5 . Del mismo modo, los porcentajes de alumnado en valores óptimos, en un rango de moderada a vigorosa intensidad, en cada uno de los tres juegos, han sido reducidos, no alcanzado el $85 \%$ del total de participantes en ninguna de las tres situaciones motrices. Además, se determina que los juegos con espacio orientado y porterías han generado mayores intensidades que el juego sin espacio orientado o mantenimiento del balón, siendo aún más elevado el esfuerzo percibido cuando se orienta el espacio y existen porterías pequeñas que cuando existen porterías grandes.

En definitiva, se constata que los juegos con espacio orientado se presentan como mejores opciones para alcanzar los niveles de intensidad recomendados, específicamente los juegos con porterías pequeñas, aunque se hace necesario un estudio en profundidad de los efectos de la modificación de los elementos estructurales de las situaciones motrices, en general, y de la orientación del espacio, en particular, sobre las demandas fisiológicas de los juegos motores.

\section{Referencias}

Albarracín, A., Moreno, J. A., y Beltrán, V. J. (2014). La situación actual de la educación física según su profesorado: Un estudio cualitativo con profesores de la Región de Murcia. CCD. Cultura, Ciencia y Deporte, 9(27), 225-234.

Baena, A., y Ruiz, P. J. (2016). El juego motor como actividad física organizada en la enseñanza y la recreación. EmásF: revista digital de educación física, (38), 73-86.

Bayer, C. (1992). La enseñanza de los juegos deportivos colectivos. Barcelona, España: H. E.

Blair, S. N. (2009). Physical inactivity: the biggest public health problem of the 21st century. British Journal of Sports Medidice, 43(1), 1-2.

Biddle, S. J. H., Sallis, J. F., \& Cavill, N. (1998).Young and active? Young people and healthenhancing physical activity: Evidence and implications. Londres: Health Education Authority.

Casamichana, D., Castellano, J., González-Morán, A., García-Cueto, H.,y García-López, J. (2011). Demanda fisiológica en juegos reducidos de fútbol con diferente orientación 
del espacio. RICYDE. Revista Internacional de Ciencias del Deporte, 7(23), 141-154.

Coquart, J., Legrand, R., Robin, S., Duhamel, A., Matran, R. y Garcin, M. (2009). Influence of successive bouts of fatiguing exercise on perceptual and physiological markers during an incremental exercise test. Psychophysiology. 46(1), 209-216.

De Hoyo, M. y Sañudo, B. (2004). Composición corporal y actividad física como parámetros desalud en niños de una población rural de Sevilla.Revista Internacional de Ciencias del Deporte, 3(6), 52-62.

De la Vega, R. (2002). Desarrollo del metaconocimiento táctico y comprensión del juego: Un enfoque constructivista aplicado al fútbol (Tesis Doctoral). Universidad Autónoma de Madrid, Madrid, España.

Gambau, V. (2015). Las problemáticas actuales de la educación física y el deporte escolar en España. Revista Española de Educación Física y Deportes, (411), 53-69.

González, I. M., Fraguela, R., y Varela, L. (2017). Niveles de actividad física en Educación Infantil y su relación con la salud. Implicaciones didácticas. Sportis: Revista TécnicoCientífica del Deporte Escolar, Educación Física y Psicomotricidad, 3(2), 358-374.

González-Arévalo, C., y Lleixà-Arribas, T. (2014). Didáctica de la Educación Física. Barcelona: GRAÓ.

Garrido, A. L. (2014). La práctica de actividad física y hábitos de salud de los futuros maestros de Educación Primaria de la Universidad de Barcelona. Un estudio sobre la incidencia de la Educación Física vivida en la ESO en su futura acción docente. (Tesis doctoral). Universidad de Barcelona, España.

Hernández-Álvarez, J. L., del-Campo-Vecino, J., Martínez-de-Haro, V \& Moya-Morales, J. M. (2010). Perception of exertion in physical education and its relationship to guidelines on physical activity. Revista Internacional de Medicina y Ciencias de la Actividad Física y el Deporte, 10(40), 609-619.

Kang, J., Hoffman, J. R., Walker, H., Chaloupka, E. C. y Utter, A. C. (2003). Regulating intensity using perceived exertion during extended exercise periods. European Journal of Applied Physiology, 89(5), 475-482.

Lago, C. (2002). La enseñanza del fútbol en edad escolar. Sevilla: Wanceulen Editorial Deportiva S. L.

Lambrick, D., Bertelsen, H., Eston, R., Stoner, L., \& Faulkner, J. (2016). Prediction of peak oxygen uptake in children using submaximal ratings of perceived exertion during treadmill exercise. European journal of applied physiology, 116(6), 1189-1195.

Lavega, P. (2007). El juego motor y la pedagogía de las conductas motrices. Revista Conexões, 5(1), 27-41.

Lobos L. L., Leyton, B., Kain, J., y Vio, F. (2013). Evaluación de una intervención educativa para la prevención de la obesidad infantil en escuelas básicas de Chile. Nutrición Hospitalaria, 28(4), 1156-1164.

Martínez, H. F. (2007). Interpretación táctica y enseñanza del fútbol. Revista digital: Lecturas: Educación Física y Deporte, (109).

Martínez, J., Contreras, O. R., Lera, A., y Aznar, S. (2012). Niveles de actividad física medido con acelerómetro en alumnos de $3^{\circ}$ ciclo de educación primaria: actividad física diaria y sesiones de educación física. Revista de Psicología del Deporte, 21(1), 117-123. 
McKenzie, T. L., Marshall, S. J., Sallis, J. F., \& Conway, T. L. (2000). Student activity levels, lesson context, and teacher behavior during middle school physical education. Research Quarterly for Exercise and Sport, 71(3), 249-259.

Mallo, J., y Navarro, E. (2008). Physical load imposed on soccer players during small-sided training games. Journal of Sports and Physical Fitness, 48(2), 166-171.

Montil, M, Barriopedro, M. I. y Oliván, J. (2005). El sedentarismo en la infancia. Los niveles de actividad física en niños/as de la Comunidad Autónoma de Madrid. Apunts. Educación Física y Deportes, 4(82), 5-11.

Nader, P. R., \& NICHD (2003). Frequency and intensity or activity of third-grade children in physical education. Archives of Pediatrics and Adolescent Medicine, 157(2), 185-190.

Naclerio, F., Barriopedro, I. y Rodríguez, G. (2008). Control de la Intensidad en los Entrenamientos de Fuerza por medio de la Percepción Subjetiva del Esfuerzo. Kronos, 8(14), 59-66.

Ozkan, A. \& Kin-Isler, A. (2007). The reliability and validity of regulating exercise intensity by ratings of perceived exertion in step dance sessions. Journal of Strength and Conditioning Research, 21(1), 296-300.

Parlebas, P. (2001). Juegos, deporte y sociedad. Léxico de praxiología motriz. Barcelona: Paidotribo.

Sanchís, I., Calahorro, F., Lara, A. J., Torres, G. (2015). Nivel de actividad física con acelerometría en clases de educación física según el nivel de práctica deportiva en alumnado de primaria. Tándem: Didáctica de la educación física, (49), 59-66.

Sierra, A. (2003). Actividad física y salud en Primaria. El compromiso fisiológico en la clase de Educación Física. Sevilla: Wanceulen Editorial Deportiva S. L.

Simons-Morton, B. G., Taylor, W. C., Snider, S. A., \& Huang, I. W. (1993). The physical activity of fifth-grade students during physical education classes. American Journal of Public Health, 83(2), 262-264.

Som, A., Zabala, M., Sánchez, E., Sánchez, C. y Ramírez, J. (2010). Conocimiento sobre frecuencia cardiaca y percepción de la intensidad del esfuerzo en alumnos universitarios de último curso de Ciencias de la Actividad Física y del Deporte. Apunts. Educación Física y Deportes, (101), 66-74.

Stratton, G. (1997). Children's heart rates during British Physical Education lessons. Journal of Teaching in Physical Education, 16(3), 357-367.

Tuñón, I., y Laíno, F. (2014). Insuficiente actividad física en la infancia: niños, niñas y adolescentes entre 5 y 17 años en la Argentina urbana. En Observatorio de la Deuda Social Argentina (Ed.), Barómetro de la Deuda Social de la Infancia (pp. 1-21). Ciudad Autónoma de Buenos Aires, Argentina: Universidad Católica Argentina.

Utter, A. C., Robertson, R. J., Green, J. M., Suminski, R. R., McAnulty, S. R. \& Nieman, D. C. (2004). Validation of the Adult OMNI Scale of perceived exertion for walking/running exercise. Medicine \& Science in Sports \& Exercise, 36(10), 1776-1780.

Villagrán, S., Rodríguez-Martín, A., Novalbos, J. P., Martínez, J. M., y Lechuga, J. L. (2010). Hábitos y estilos de vida modificables en niños con sobrepeso y obesidad. Nutrición Hospitalaria, 25(5), 823-831.

WHO. World Health Organization. (2010). Global recommendations on physical activity for health. Geneva: World Health Organization. 
Yelling, M., Lamb, K. L. \& Swaine, I. L. (2002). Validity of a pictorial perceived exertion scale for effort estimation and effort production during stepping exercise in adolescent children. European Physical Education Review, 8(2), 157-175. 